\title{
Cambio climático y su impacto potencial en la ocurrencia de incendios forestales en la zona centro-sur de Chile $\left(33^{\circ}-42^{\circ} \mathrm{S}\right)$
}

\author{
Climatic change and its potential impact on forest fire occurrence in \\ south-central Chile $\left(33^{\circ}-42^{\circ} \mathrm{S}\right)$ \\ Mauro E González ${ }^{\text {a*}}$, Antonio Lara ${ }^{\mathrm{a}, \mathrm{b}}$, Rocío Urrutia ${ }^{\mathrm{c}, \mathrm{b}}$, Juvenal Bosnich ${ }^{\mathrm{d}}$ \\ *Autor de correspondencia: ${ }^{\text {a }}$ Universidad Austral de Chile, Facultad de Ciencias Forestales y Recursos Naturales, Instituto de \\ Silvicultura, Laboratorio Ecología de Bosques, casilla 567, Valdivia, Chile, maurogonzalez@uach.cl \\ ${ }^{b}$ Fundación Centro de los Bosques Nativos Forecos, Valdivia, Chile. \\ ${ }^{c}$ Environmental Change Institute, School of Geography and the Environment, University of Oxford, Oxford, UK. \\ ${ }^{\mathrm{d}}$ Corporación Nacional Forestal, Departamento de Protección Forestal, región de Los Lagos, Puerto Montt, Chile.
}

\begin{abstract}
SUMMARY
Fire frequency and severity have increased in different regions of the world. The main causes explaining these new fire patterns would be associated with climate and land-use change. The present contribution briefly examines the recent changes of climate and predictions and their potential influence on fire activity in south-central Chile, along with the necessary adaptation measures to cope with this problem. Different studies coincide in that main effects of climate change in this area will be associated with lower precipitations which would result in an increment of the occurrence and area affected by forest fires. The major and more urgent challenge is defining the proper policies and strategies of mitigation and adaptation to promote healthy forests (i.e., productive, diverse and resilient), ensuring a continuum provision of ecosystem services under a changing climate.
\end{abstract}

Key words: forest diversification, plan forest adaptation, resilience, fire regime.

\section{RESUMEN}

En muchas regiones del mundo los incendios están siendo cada vez más frecuentes y severos. Las principales causas de estos patrones se asociarían tanto a cambios en el clima como en las prácticas de uso de la tierra. La presente contribución examina sucintamente los recientes cambios y proyecciones climáticas y su potencial influencia en la ocurrencia de incendios en la zona centro-sur de Chile, así como las medidas de adaptación necesarias para hacer frente a este problema. Diversos estudios coinciden en que el mayor efecto del cambio climático en esta zona estará asociado a la disminución de las precipitaciones lo cual incidiría en un incremento de la ocurrencia y área afectada por incendios forestales. Bajo este escenario, el principal desafío está en definir acertadamente las políticas y estrategias de mitigación y adaptación sectoriales con la finalidad de promover bosques más saludables, -en términos de productividad, diversidad y resiliencia- asegurando una provisión continua de servicios ecosistémicos bajo un clima cambiante.

Palabras clave: diversificación forestal, plan de adaptación forestal, resiliencia, régimen de incendio.

\section{INTRODUCCIÓN}

Durante las últimas décadas, incendios, plagas y sequías se han intensificado en frecuencia y severidad en muchas regiones del mundo. En Estados Unidos el área forestal afectada por incendios ha aumentado seis veces en las últimas dos décadas (Mann y Kump 2009). En el oeste de ese país, el aumento repentino y marcado de la frecuencia y duración de incendios de gran magnitud se asocia a los recientes cambios climáticos en la región (Westerling et al. 2006). Un cambio abrupto hacia primaveras inusualmente cálidas, reducidos montos de precipitación invernal, derretimiento temprano de la nieve en primavera y veranos más secos y prolongados son señaladas como las causas principales de estos patrones en el régimen de incendios (Westerling et al. 2006). Similarmente, en el oeste de Nor- teamérica, se ha observado a una escala sin precedentes ( $>13$ millones de hectáreas sólo en Columbia Británica) el ataque de insectos de la corteza (Dendroctonus ponderosae Hopkins y Dendroctonus rufipennis Kirby) a bosques de Picea glauca (Moench) Voss. y Pinus contorta Douglas ex Loudon. Veranos inusualmente más cálidos e inviernos menos rigurosos favorecerían los ciclos de vida de los insectos (Raffa et al. 2008, Konkin y Hopkins 2009). En Canadá, diversos estudios anticipan un rápido y profundo impacto del cambio climático en la frecuencia, extensión y severidad de los incendios en los bosques boreales (Webber y Flannigan 1997, Bergeron et al. 2004). En amplias zonas de Europa, el fuego es el principal modelador del paisaje (Pausas et al. 2008), con un claro aumento de su frecuencia y extensión en los últimos 50 años (Moreno et al. 1998). Recientemente, en distintas partes del mundo 
se ha observado una mayor tasa de mortalidad arbórea y mortalidades episódicas, a lo cual contribuirían importantemente el aumento de la temperatura y déficit hídrico (van Mantgem et al. 2009, Allen et al. 2010). Este potencial aumento en la tasa de mortalidad es de especial preocupación debido a las interacciones con otros procesos ecosistémicos mediados climáticamente como son plagas e incendios.

Dentro de este contexto, los objetivos centrales del presente trabajo son examinar sucintamente el potencial efecto de los cambios climáticos proyectados para Chile sobre los patrones de ocurrencia de incendios e identificar los desafíos y acciones clave para promover un paisaje forestal de mayor resiliencia frente al actual escenario climático previsto.

\section{INCENDIOS FORESTALES EN EL CONTEXTO DEL CAMBIO CLIMÁTICO EN CHILE}

En el centro-sur de Chile como también en el norte de la Patagonia argentina $\left(38^{\circ}-43^{\circ} \mathrm{S}\right)$, la ocurrencia de incendios se asocia estrechamente a condiciones en el Pacífico tropical (El Niño Oscilación del Sur, ENOS) y a variaciones en los patrones de circulación atmosférica en latitudes medias y altas (Kitzberger y Veblen 2003, Veblen et al. 1999, González y Veblen 2006) que influencian la precipitación y temperatura.

En Chile, como en otras partes del mundo, se estima que las temperaturas y las precipitaciones presentarán cambios importantes. En un estudio sobre variabilidad climática en el siglo XXI (CONAMA 2006), se estimó que hacia finales de siglo la zona centro-sur de Chile $\left(31^{\circ}-45^{\circ} \mathrm{S}\right)$ experimentaría una disminución en las precipitaciones de hasta un 25 y $40 \%$ en el periodo de primavera y verano, respectivamente. Estas proyecciones vendrían a profundizar el patrón actual de disminución en las precipitaciones, las que han decrecido entre 20 y $40 \%$ especialmente en el centro-sur del país (1901-2005, Trenberth et al. 2007). En relación a los escenarios esperados para la temperatura, se espera un calentamiento en todas las regiones, el que variaría entre 2 y $4{ }^{\circ} \mathrm{C}$ en el escenario más severo para fines del siglo XXI (CONAMA 2006).

Recientes estudios dendroclimáticos desarrollados en la zona central de Chile $\left(33^{\circ}-37^{\circ} \mathrm{S}\right)$ han dado cuenta de un incremento significativo en la recurrencia de las sequías y disminución de las precipitaciones, así como de una mayor recurrencia de caudales menores a la media durante el siglo XX, comparado con siglos previos (Le Quesne et al. 2006, Christie et al. 2010, Urrutia et al. 2011). Esto contribuiría, dadas las condiciones ambientales más favorables, a incendios más frecuentes y de mayor extensión.

En la región centro-sur de Chile, las estadísticas muestran un aumento consistente y significativo en el número de incendios forestales - $99 \%$ de origen antrópico- durante las últimas tres décadas (figura 1A). Por otra parte, en la zona central de Chile se ha registrado un área quemada particularmente alta asociada a la ocurrencia de fenómenos severos de El Niño (figura 1B). A esta latitud (32-35 $\mathrm{S}$ ), donde predomina una vegetación arborescente y abierta -tipo esclerófila-, inviernos y primaveras más lluviosos en años Niño promoverían la acumulación de combustible generando condiciones para la ignición y propagación de incendios, no en la temporada inmediata de incendio sino en la temporada siguiente. En algunos casos, esta acumulación de combustible durante años Niño estaría acompañada al año siguiente de la ocurrencia de condiciones más secas de lo normal asociadas al fenómeno de La Niña, como en los años 1988 y 1998, lo que resultaría en una mayor superficie quemada durante la temporada de incendios. En este contexto, es importante señalar que el fenómeno del Niño ha aumentado su variabilidad en términos de frecuencia y amplitud durante las últimas décadas (Yang y Zhang 2008), como es demostrado por los eventos de 1982/1983 y 1997/1998, los eventos más fuertes del siglo $\mathrm{XX}$, que contribuyeron a que esas décadas fueran las más cálidas del siglo pasado.

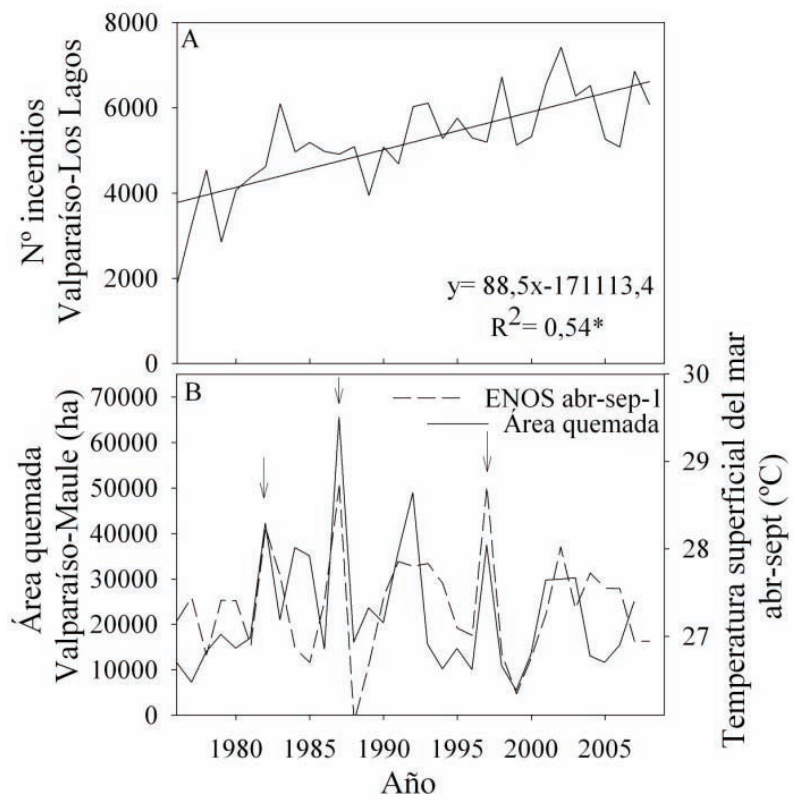

Figura 1.A) Número de incendios entre las regiones de Valparaíso y Los Lagos para el período 1976-2008. B) Relación entre el área total afectada por incendios y la temperatura superficial del mar (abril-septiembre (-1)) en la región El Niño Oscilación del Sur 3.4 (ENOS 3.4) en Chile central (regiones de Valparaíso a Maule). Las flechas indican los tres años de eventos El Niño más intensos en este período (1982-83, 1987-1988, 1997-98). Fuentes: CONAF (2009) y NOAA (2010).

A) Number of fires between Valparaíso and Los Lagos regions for the period 1976-2008. B) Relationship between total area affected by fire in these regions and sea surface temperature (April - September $(-1))$ for the region ENSO 3.4 in central Chile (regions of Valparaíso to Maule). Arrows indicate the three stronger El Niño events in this period (1982-83, 1987-1988, 1997-98). Sources: CONAF (2009) and NOAA (2010). 
En el caso particular de los incendios que han afectado plantaciones de especies exóticas, desde la década de 1990 se ha observado una tendencia al aumento de las temporadas de incendio extremas en términos de la superficie anualmente quemada (figura 2). Esta tendencia, además de reflejar el aumento de la superficie base de plantaciones desde la década de 1970, se debería en buena medida a la mayor superficie quemada principalmente en la región del Biobío -que concentra cerca del $40 \%$ de la superficie nacional de plantaciones- con dos temporadas que superaron 20.000 ha (1998-99 y 2006-07). Por otra parte, durante varios años la temporada de incendios ha experimentado mayor duración en toda esta macroregión (regiones de Valparaíso a Los Lagos) debido, entre otros factores, al alargamiento del déficit hídrico estival o a la disminución de las precipitaciones en invierno (CONAF 2009).

\section{PLAN DE ADAPTACIÓN FORESTAL: UN DESAFÍO CLAVE PARA EL SIGLO XXI}

Como en otras regiones, el cambio climático en Chile no es teórico y sus efectos comienzan a advertirse. El escenario climático proyectado para las próximas décadas de disminución pronunciada de las precipitaciones y el aumento de la recurrencia de sequías, resultaría en un incremento en la ocurrencia (número) y el área afectada por incendios (figura 3). Particularmente vulnerables a estos cambios en el régimen de incendios estarían aquellas regiones dominadas por extensas plantaciones y ecosistemas remanentes altamente fragmentados e invadidos por especies exóticas, derivado principalmente del tipo, homogeneidad y continuidad del combustible.

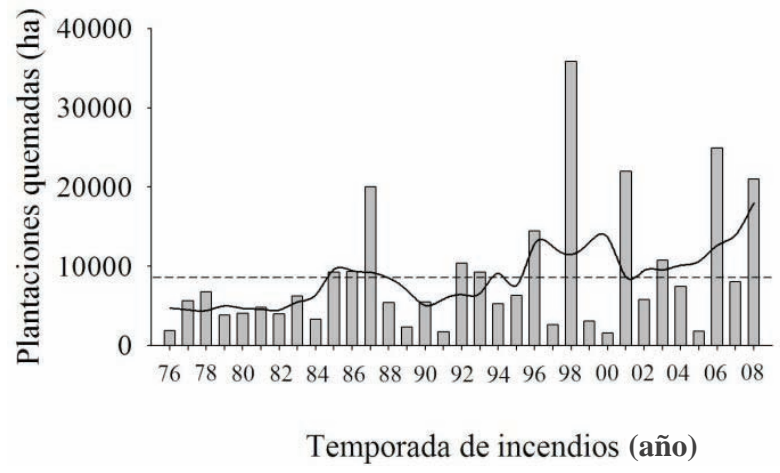

Figura 2. Superficie de plantaciones quemadas por temporada de incendios entre las regiones de Valparaíso y La Araucanía en el período 1976-2008. La línea segmentada indica el promedio de superficie afectada (8.620 hectáreas al año) para el periodo. La curva es una media móvil de cinco años del valor respectivo.

Forest plantations burned in each fire season between Valparaíso and Araucanía region for the period 1976-2008. The segmented line indicates average burned area $(8,620 \mathrm{ha})$ for the period. The curve is a 5 -year moving average of the respective value.
Figura 3. Diagrama conceptual que representa el rango de variabilidad del clima actual y del régimen de incendios (ocurrencia y área) y los cambios esperados en éste último como resultado del aumento de sequías y temperatura asociadas con el cambio climático proyectado (modificado de Allen et al. 2010).

Conceptual diagram that represents the current range of climate variability and fire regime (occurrence and area) and expected changes of the latter resulting from the increase of drought and temperature associated with projected climate change (modified from Allen et al. 2010).

Desde 1996, Chile viene abordando el tema del cambio climático a través del desarrollo de la Estrategia Nacional de Cambio Climático y el Plan de Acción Nacional de Cambio Climático (PANCC; 2008-2012), cuyo objetivo principal es minimizar los impactos adversos de dichos cambios mediante acciones integradas que permitan determinar la vulnerabilidad del país y las medidas de adaptación para enfrentarlos adecuadamente (Neuenschwander 2010). Por otra parte, el Ministerio de Agricultura, en mayo 2008, constituyó el Consejo del Cambio Climático y Agricultura, compuesto por destacadas personalidades de los sectores productivo, público y académico, con la misión de apoyar al ministerio en la definición de los principales lineamientos y prioridades a considerar en un programa de adaptación y mitigación del cambio climático en el ámbito silvoagropecuario.

Una de las principales prioridades para hacer frente a los factores de estrés más críticos sobre los bosques incendios, propagación de plagas y especies invasoras derivados de las predicciones de cambio climático es el desarrollo de un "plan de adaptación forestal" como parte del PANCC. Entre las acciones clave a ser impulsadas dentro de este plan está la diversificación del recurso forestal. La masificación y coalescencia de las plantaciones 
forestales de Pinus radiata D. Don y Eucalyptus sp., en muchas comunas desde la región de Valparaíso a la de Los Lagos, las colocan en una situación de alta vulnerabilidad tanto a incendios como al ataque por plagas y enfermedades. Además, ante el escenario de una mayor probabilidad de incendios en paisajes dominados por plantaciones se puede esperar un severo impacto en ecosistemas naturales insertos en esta matriz. Especialmente vulnerables serán los pequeños fragmentos de bosque nativo ubicados en las regiones del Maule y Biobío donde aún sobreviven poblaciones de especies endémicas en peligro (ejemplo: Nothofagus alessandri Espinosa, Pitavia punctata (R. et P.) Mol., Gomortega queule (Mol.) Baillon, Berberidopsis coralina Hook. f.). En este contexto, la diversificación implicaría con la pertinente coordinación entre las instituciones del Estado- fomentar el desarrollo y mantención de diversas actividades y usos del territorio dentro de la matriz de plantaciones (ejemplo: praderas, uso agrícola, manejo bosque nativo, turismo), valorando en forma adecuada los servicios ecosistémicos asociados.

Además de propender a un territorio más diversificado en términos de la matriz productiva -asegurando a la vez la adecuada representación de ecosistemas naturales- los principales requerimientos apuntan esencialmente a mejorar aspectos de planificación, diseño y prácticas forestales. Un mosaico de rodales contiguos de edades distintas, respetando zonas de protección y parches de vegetación nativa, además de favorecer aspectos estéticos y de conectividad, contribuiría a un menor riesgo y propagación de incendios. Estos aspectos de diseño implicarían talas rasas de rodales coetáneos de menor superficie contribuyendo, a su vez, a disminuir los impactos ecológicos y visuales de estas intervenciones.

Dentro de este contexto, la propuesta surgida a fines del 2008 desde la Corporación Chilena de la Madera de aumentar en un millón de hectáreas la superficie de plantaciones monoespecíficas entre las regiones del Maule y La Araucanía (CORMA 2010), parece absolutamente inconveniente y discordante ante las predicciones de cambio climático. Esta propuesta no haría más que incrementar la vulnerabilidad de las plantaciones a los incendios forestales agravando a su vez el problema de escasez de agua que ya sufren distintas localidades en comunas del centro-sur del país. Esta expansión, sin duda, iría aparejada al mayor gasto de recursos que el Estado y empresas privadas deberán destinar al combate de incendios forestales, que a juicio de expertos ha crecido durante los últimos años a cifras estimadas en más US\$ 30 millones anuales, sin considerar las pérdidas directas.

El sector forestal en Chile y en el mundo se encuentra ante un cambio de paradigma en el manejo y prácticas asociadas a las plantaciones productivas de rápido crecimiento. Esto implica importantes desafíos políticos, institucionales y culturales a los cuales tanto el Estado como las empresas ya comienzan a adaptarse. Recientes iniciativas internacionales como las plantaciones de nueva generación (New Generation Plantations) y principios de certificación como los definidos por la organización Forest Stewarship Council (FSC) son ejemplos que apuntan a promover y normar el buen manejo y sustentabilidad de los bosques y plantaciones. En este contexto, la principal responsabilidad del Estado radica en promover políticas pertinentes que apunten, en lo general, al ordenamiento del territorio y, en lo particular, a la diversificación del sector forestal, generando conjuntamente la tan urgente institucionalidad sectorial capaz de enfrentar y conducir los desafíos impuestos por el cambio climático y la sociedad. Estas medidas constituyen el primer paso para una efectiva adaptación frente al cambio climático, contribuyendo tanto a reducir el riesgo de incendios como a promover bosques más saludables -en términos de productividad, diversidad y resiliencia- en el largo plazo.

\section{AGRADECIMIENTOS}

Universidad Austral de Chile (DID-I-2010-5), CRN II 2047 del Instituto Interamericano para la Investigación del Cambio Global (IAI). Además, estos resultados han recibido fondos del Séptimo Programa Marco de la Unión Europea (FP7/2007- 2013) bajo el proyecto $n^{\circ} 243888$.

\section{REFERENCIAS}

Allen CD, AK Macalady, H Chenchouni, D Bachelet, N McDowell, M Vennetier, T Kitzberger, A Rigling, DD Breshears, EH Hogg, P Gonzalez, R Fensham, Z Zhangm, J Castro, N Demidova, J Lim, G Allard, SW Running, A Semerci, N Cobb. 2010. A global overview of drought and heat-induced tree mortality reveals emerging climate change risks for forests. Forest Ecology and Management 259(4): 660-684.

Bergeron Y, M Flannigan, S Gauthier, A Leduc, P Lefort. 2004. Past, current and future fire frequency in the Canadian boreal forest: Implications for sustainable forest management. Ambio 33(6): 356-360.

Christie D, J Boninsegna, M Cleaveland, A Lara, C Le Quesne, M Morales, M Mudelsee, D Stahle, R Villalba. 2010. Aridity changes in the Temperate-Mediterranean transition of the Andes since AD 1346 reconstructed from tree-rings. Climate Dynamics DOI 10.1007/s00382-009-0723-4.

CONAF (Corporación Nacional Forestal, CL). 2009. Estadísticas de Incendio. Consultado 26 dic. 2009. Disponible en http:// www.conaf.cl/??page $=$ home/contents\&seccion_id=322 af2 5bd24d696f5abe1 ed700dc5828\&unidad $=8 \&$ pagina $=$

CONAMA (Comisión Nacional de Medio Ambiente, CL). 2006. Estudio de la variabilidad climática en Chile para el siglo XXI. Informe Final. Santiago, Chile. CONAMA. 63 p.

CORMA (Corporación Chilena de la Madera, CL). 2010. Proyecto forestación de 1 millón hectáreas erosionadas. Acuerdo del Bío-Bío. Concepción, Chile. CORMA. 15 p.

González ME, TT Veblen. 2006. Climatic influences on fire in Araucaria araucana-Nothofagus forests in the Andean cordillera of south-central Chile. Ecoscience 13(3): 342-350.

Kitzberger T, TT Veblen. 2003. Influences of climate on fire in 
Northern Patagonia, Argentina. In Veblen TT, WL Baker, G Montenegro, TW Swetnam eds. Fire regimes and climatic change in temperate ecosystems of the western Americas. Springer-Verlag. p. 296-321.

Konkin D, K. Hopkins. 2009. Aprender cómo hacer frente a las perturbaciones derivadas del cambio climático y a otros fenómenos catastróficos que afectan a los bosques. Unasylva 60(231/232): 17-23.

Le Quesne C, DW Stahle, MK Cleaveland, MD Therrell, JC Aravena, J Barichivich. 2006. Ancient Austrocedrus tree-ring chronologies used to reconstruct central Chile precipitation variability from A.D. 1200 to 2000. Journal of Climate 19(22): 5731-5744.

Mann ME, LR Kump. 2009. Dire predictions: understanding global warming. New York, USA. DK publishing. 208 p.

Moreno JM, A Vázquez, R Vélez. 1998. Recent history of forest fires in Spain. In Moreno JM ed., Large Forest Fires. Leiden, The Netherlands. Backhuys Publishers. p. 159-185.

Neuenschwander A. 2010. El Cambio Climático en el Sector Silvoagropecuario de Chile. Fundación para la Innovación Agraria. Santiago, Chile. Ministerio de Agricultura. 123 p.

NOAA (National Oceanic and Atmospheric Administration, US). 2010. Niño 3.4 Index. Consultado 10 may. 2010. Disponible en http://www.esrl.noaa.gov/psd/data/correlation/ nina34.data

Pausas JG, J Llovet, A Rodrigo, R Vallejo. 2008. Are wildfires a disaster in the Mediterranean basin? - A review. International Journal of Wildland Fire 17(6): 713-723.

Raffa KF, BH Aukema, BJ Bentz, AL Carroll, JA Hicke, MG Turner, WH Romme. 2008. Cross-scale drivers of natural disturbances prone to anthropogenic amplification: the dynamics of bark beetle eruptions. Bioscience 58(6): 501-517.
Trenberth KE, PD Jones, P Ambenje, R Bojariu, D Easterling, A Klein Tank, D Parker, F Rahimzadeh, JA Renwick, M Rusticucci, B Soden, P Zhai. 2007. Observations: Surface and Atmospheric Climate Change. In Solomon S, D Qin, M Manning, Z Chen, M Marquis, KB Averyt, M Tignor, HL Miller. Climate Change 2007: The Physical Science Basis. Contribution of Working Group I to the Fourth Assessment Report of the Intergovernmental Panel on Climate Change. Cambridge, United Kingdom. Cambridge University Press. p. 235-336.

Urrutia RB, A Lara, R Villalba, DA Christie, C Le Quesne, A Cuq. 2011. Multi-century tree-ring reconstruction of annual streamflow for the Maule River watershed in South-Central Chile.Water Resources Research. DOI:10.1029/2010WR009562.

van Mantgem PJ, NL Stephenson, JC Byrne, LD Daniels, JF Franklin, PZ Fulé, ME Harmon, AJ Larson, JM Smith, AH Taylor, TT Veblen. 2009. Widespread Increase of Tree Mortality Rates in the Western United States. Science 323 (5913): 521-524.

Veblen TT, T Kitzberger, R Villalba, J Donnegan. 1999. Fire history in northern Patagonia: the roles of humans and climatic variation. Ecological Monographs 69(1): 47-67.

Weber MG, MD Flannigan. 1997. Canadian boreal forest ecosystem structure and function in a changing climate: Impacts on fire regimes. Environmental Reviews 5(3-4): 145-166.

Westerling AL, HG Hidalgo, DR Cayan, TW Swetnam. 2006. Warming and earlier spring increase western U.S. forest wildfire activity. Science 313(5789): 940-943.

Yang H, Q Zhang .2008. Anatomizing the ocean's role in ENSO changes under global warming. Journal of Climate 21(24): 6539-6555. 
